\title{
Diabetes tipo 2 no idoso, apresentação clínica e metas de tratamento
}

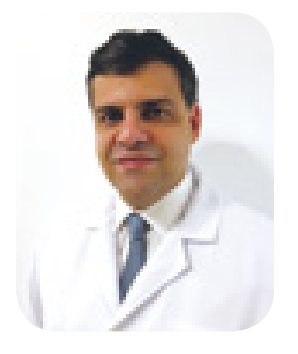

\section{RESUMO}

O diabetes é uma doença cuja prevalência vem crescendo ao longo das últimas décadas. Estudos epidemiológicos têm evidenciado uma relação entre idade e o desenvolvimento da doença, sendo estimada uma frequência aproximada de $25 \%$ nos idosos. Os idosos com diabetes precisam ser avaliados de forma individualizada, uma vez que a apresentação clínica é heterogênea e pode variar entre dois extremos, que incluem desde indivíduos saudáveis, autônomos e independentes até aqueles mais frágeis, com lesões de órgão-alvo, nos quais o processo de senilidade se mostra mais intenso. Evidências têm falhado em demonstrar benefício com o tratamento mais agressivo, buscando uma meta glicêmica muito baixa nos indivíduos idosos, com comorbidades, déficit cognitivo e perda de capacidade funcional. É preciso que essa população seja abordada de forma diferenciada, devendo sempre individualizar cada paciente de acordo com sua capacidade funcional, autonomia, perfil social, presença de doenças associadas e fatores de risco para hipoglicemia, a fim de se proporcionar tratamento adequado. Dadas as evidências atuais, a meta glicêmica deve sempre ser estabelecida de forma a evitar desfechos negativos.

PALAVRAS-CHAVE: diabetes no idoso, metas glicêmicas, controle do diabetes.

\section{EPIDEMIOLOGIA}

O diabetes é uma doença cuja prevalência vem crescendo ao longo das últimas décadas. De acordo com dados da International Diabetes Federation, estima-se que existem aproximadamente 425 milhões de diabéticos com idades entre 20 e 79 anos no mundo. Entre esses, $79 \%$ vivem nos países em desenvolvimento, sendo que, no Brasil, a doença acomete cerca de 14 milhões de indivíduos. ${ }^{1}$

\author{
Jonas Gordilho Souza ${ }^{1}$
}

Dentre os fatores que contribuem para esse crescimento, destacam-se o envelhecimento populacional, urbanização, sedentarismo, obesidade e erro alimentar, que são características da sociedade contemporânea. ${ }^{2,3}$ Estudos epidemiológicos têm evidenciado uma relação direta entre idade e o desenvolvimento da doença, sendo estimado, atualmente, um número de 122,8 milhões de idosos diabéticos, com idades entre 65 e 99 anos, em todo o mundo. ${ }^{1}$ No Brasil, é observada uma prevalência de $22,1 \%$ nos indivíduos com idade maior ou igual a 65 anos, o que corresponde a um número 18,4 vezes maior quando comparado aos jovens de 25 a 34 anos. ${ }^{4}$

\section{CLASSIFICAÇÃO}

Existem diferente tipos de distúrbios metabólicos que podem culminar com um aumento dos níveis glicêmicos. Eles podem ser resultado tanto de defeitos na ação quanto na secreção de insulina. ${ }^{5}$ A classificação sugerida pela Associação Americana de Diabetes (ADA) inclui os subtipos: diabetes tipo 1, diabetes tipo 2, diabetes gestacional e outros tipos específicos (diabetes latente autoimune do adulto - LADA, mature onset diabetes ofyoung - MODY, defeitos genéticos funcionais da célula beta ou na ação da insulina, diabetes secundária a doenças pancreáticas, medicamentosa e por outras doenças endocrinológicas (Cushing, Feocromocitoma, acromegalia, glucagonoma) ${ }^{6}$

O diabetes tipo 1, que acomete o indivíduo em idades mais jovens e que corresponde de 5 a $10 \%$ dos casos, é caracterizado pela destruição autoimune das células beta no pâncreas, levando a uma incapacidade absoluta na secreção de insulina. ${ }^{6}$ Em função da melhoria na qualidade do tratamento e aumento da longevidade desses pacientes, tem-se observado cada vez mais indivíduos idosos com diabetes tipo $1 .^{1}$

O diabetes tipo 2, que é a forma mais comum observada, é resultante de uma associação entre resistência 
periférica à insulina e deficiência na sua secreção. Esse subtipo se caracteriza por uma perda progressiva na capacidade de produção de insulina ao longo dos anos, o que pode levar o indivíduo a ter uma deficiência absoluta, com um comportamento semelhante ao do diabetes tipo $1 .{ }^{6}$ Uma vez que esta é a forma mais comum observada nos idosos, será o principal foco desta revisão.

\section{DIABETES TIPO 2 NO IDOSO}

Ao avaliar o idoso com diabetes, existem algumas peculiaridades que devem ser destacadas, uma vez que a apresentação clínica é heterogênea e pode variar entre dois extremos, que incluem desde indivíduos saudáveis, autônomos e independentes, com poucas complicações, até aqueles mais frágeis, com lesões de órgão-alvo, nos quais o processo de senilidade se mostra mais agressivo. ${ }^{7}$ A síndrome de fragilidade é uma condição frequente, observada em cerca de 5 a $35 \%$ dos idosos acima de 65 anos. ${ }^{8}$ Ela é caracterizada pela presença de sarcopenia, alteração endocrinológica e imunológica, o que leva a uma maior vulnerabilidade aos estressores ambientais. Fried et. al., 2001, descreveram um modelo fenotípico que inclui baixa força de preensão palmar, fadiga, perda de peso, baixa de gasto energético e baixa velocidade de marcha. Uma vez que a síndrome de fragilidade representa uma perda de capacidade funcional, que pode ser exacerbada por complicações de doenças crônicas e agudas, é esperado um maior risco nos indivíduos diabéticos com complicações micro e macrovasculares.

Entre outras particularidades que ocorrem na apresentação clínica do diabetes no idoso, destacam-se as síndromes geriátricas como a depressão, demência, polifarmácia, quedas, déficts sensoriais e incontinência. A seguir serão descritos alguns aspectos mais relevantes destas síndromes. ${ }^{7}$

A depressão é uma condição comumente observada na terceira idade e tem uma prevalência aumentada no diabetes. ${ }^{9}$ Devido à possibilidade de existir uma associação entre sintomas de humor e controle glicêmico inadequado é importante que a depressão seja sempre rastreada. Frequentemente, os pacientes deprimidos cursam com desmotivação e perda de autocuidado, que são aspectos fundamentais para o sucesso terapêutico no diabetes. ${ }^{10}$

As demências de Alzheimer e vascular também são observadas com uma maior frequência no idoso com diabetes. ${ }^{11}$ Esses indivíduos podem ter apresentações que variam desde um distúrbio cognitivo leve, com alterações em funções executivas e memória, até quadros mais graves de síndromes demenciais avançadas. É importante rastrear alterações cognitivas em todos os idosos com diabetes, uma vez que essas dificuldades podem interferir de forma negativa na adesão ao tratamento farmacológico e não farmacológico. Além disso, os alvos de tratamento podem ser estabelecidos de forma individualizada, a depender do grau de alteração cognitiva de cada paciente. ${ }^{6}$

Com a senescência, ocorrem mudanças no organismo que interferem nos processos de farmacocinética e farmacodinâmica, tornando-os mais vulneráveis aos efeitos adversos de fármacos. ${ }^{12,13,14}$ Essa vulnerabilidade pode, ainda, ser agravada pela polifarmácia, que é definida como a presença de 5 ou mais medicações. ${ }^{15}$ Acredita-se que o diabetes seja um grande fator de risco para a presença de polifarmácia, já que esses pacientes costumam apresentar comorbidades e necessitam de tratamentos, que se tornam mais complexos com o passar dos anos e com a progressão da falência pancreática. Muitos precisam lidar com rotinas que incluem o uso de múltiplas doses de insulina, o que demanda um conhecimento sobre o tempo de ação da droga, e reconhecimento dos sinais e sintomas de hipoglicemia. ${ }^{16}$ Já é conhecido que a presença de polifarmácia aumenta o risco de reações adversas, interações medicamentosas e desfechos negativos, como internações, mortalidade e quedas. ${ }^{17,18} \mathrm{Em}$ virtude desses achados, é importante ponderar o uso de medicações desnecessárias em idosos com diabetes.

Alguns autores vêm também estudando a relação entre o diabetes e quedas, uma vez que os fatores de risco intrínsecos mais comumente envolvidos com esse desfecho podem estar presentes (perda de visão e audição, neuropatia periférica, incontinência urinária, hipotensão postural e polifarmácia). Outra condição que contribui para o risco de quedas é a hipoglicemia. ${ }^{19,20}$ Acredita-se que essa vulnerabilidade para hipoglicemia seja exacerbada pela presença de condições como: diminuição da taxa de filtração glomerular, alimentação inadequada, uso de secretagogos e insulina. ${ }^{21} E$ importante que familiares e cuidadores sejam bem informados quanto ao risco de hipoglicemia, uma vez que, frequentemente, os idosos se apresentam com delirium, tontura e fraqueza, sem manifestar sintomas adrenérgicos, como tremores e sudorese, o que torna mais difícil o diagnóstico. ${ }^{22} \mathrm{~A}$ hipoglicemia deve ser evitada a todo custo, já que pode resultar em complicações clínicas relevantes, como perda cognitiva, eventos cardiovasculares e óbito. ${ }^{23}$

\section{CRITÉRIOS DIAGNÓSTICOS}

Embora existam diferenças na apresentação do diabetes entre os indivíduos jovens e idosos, os critérios 
diagnósticos são semelhantes: glicemia de jejum $\geq 126$ $\mathrm{mg} / \mathrm{dL}$, hemoglobina glicada $(\mathrm{HbA} 1 \mathrm{c}) \geq 6,5 \%$, glicemia ao acaso $\geq 200 \mathrm{mg} / \mathrm{dL}$ (associados a sintomas de hiperglicemia) e teste de tolerância oral de glicose com glicemia após 2 horas de $200 \mathrm{mg} / \mathrm{dL}$, repetidos para confirmação se não houver sintomas compatíveis. ${ }^{6}$ É importante, no entanto, considerar os erros que envolvem o método de leitura da $\mathrm{HbA1C}$, que pode ser alterado por algumas condições, a exemplo da anemia, uremia, tireoideopatia, entre outras. ${ }^{24}$

\section{METAS GLICÊMICAS E PECULIARIDADES NO TRATAMENTO}

Diversos estudos já foram realizados com o objetivo de avaliar o efeito do controle glicêmico na redução das lesões de órgão-alvo. Infelizmente, não existem estudos robustos feitos especificamente para indivíduos idosos, em especial aqueles acima de 75 anos. Sendo assim, as evidências atuais se apoiam em subanálises de trabalhos realizados com populações de diversas faixas etárias.

O United Kingdom Prospective Diabetes (UKPDS) foi o primeiro estudo de grande impacto com evidência de que o controle glicêmico intensivo, objetivando um valor de $\mathrm{HbA1C}$ menor que $7 \%$, leva a uma redução na incidência de complicações microvasculares. ${ }^{25}$ Nesse estudo foram randomizados 3.867 indivíduos em dois grupos, sendo um tratado com antidiabéticos orais ou insulina e outro grupo cujo único tratamento foi uma dieta orientada. Depois de 10 anos de seguimento, observou-se uma redução de $25 \%(7-40, p<0,001)$ para as complicações microvasculares no grupo tratamento intensivo [HbA1C média 7,0\% $(6 \cdot 2-8 \cdot 2)$ ], comparado ao grupo dieta $[\mathrm{HbA1C}$ média $7,9 \%(6 \cdot 9-8 \cdot 8)]$.

Posteriormente, após a conclusão do UKPDS, foi realizado um seguimento post-trial, no qual 3.277 participantes foram monitorados por mais 10 anos, sendo que, dessa vez, cada indivíduo era tratado de maneira individualizada pelo seu médico. Após esse período de acompanhamento, observou-se uma persistência na redução dos desfechos microvasculares de $24 \%$ ( $p=$ $0,001)$, além do aparecimento de uma redução no risco de infarto do miocárdio de $15 \%(p=0,01)$ e redução de morte por qualquer causa em $13 \%(p=0,007)$. É importante ressaltar que esses resultados foram evidenciados mesmo sem haver diferença nos valores de $\mathrm{HbA} 1 \mathrm{C}$ entre os dois grupos. A partir de então, foi introduzido o conceito de que a memória metabólica do controle glicêmico adequado nos momentos iniciais da doença poderia levar a um benefício permanente na redução nas lesões de órgãos-alvo associadas. ${ }^{26}$
Apesar dos resultados encontrados no UKPDS, devemos ressaltar que nele foi avaliada uma amostra de indivíduos com uma idade mediana de 54 anos (48-60), com baixa incidência de complicações cardiovasculares no início do seguimento, o que não permite extrapolar os resultados obtidos aos idosos com doença mais grave e com maior prevalência de complicações cardiovasculares.

Foram, então, realizados novos estudos que avaliaram sujeitos mais idosos, com doença cardiovascular estabelecida e maior tempo de diabetes, sem que fosse demonstrado qualquer benefício do controle glicêmico intensivo nesse grupo. ${ }^{27,28,29}$ Ao contrário das expectativas, em um dos estudos, foi observada uma maior mortalidade nos participantes com valores de $\mathrm{HbA1C}$ menores que 6,0\%. ${ }^{27}$ Nesse estudo foram avaliados 10.251 diabéticos, com idade média de 62,2 anos, $\mathrm{HbA} 1 \mathrm{C}$ média de $8,1 \%$, sendo que $35 \%$ já haviam sofrido evento cardiovascular prévio. Após o recrutamento, os participantes foram randomizados para 2 grupos: 0 primeiro com alvo de $\mathrm{HbA} 1 \mathrm{C}$ menor que $6,0 \%$; o segundo grupo-controle com alvo de $\mathrm{HbA1C}$ entre 7,0 e $7,9 \%$. Após 3,5 anos de seguimento, o estudo teve que ser interrompido em função de um aumento de $22 \%$ na mortalidade do grupo com tratamento intensivo, sendo que não foi observada uma redução em eventos cardiovasculares maiores.

De acordo com as evidências atuais, parece existir uma curva em $U$ para o controle glicêmico no idoso, na qual os extremos que incluem hipoglicemia e hiperglicemia estão associados à maior mortalidade e desfechos negativos. Neste contexto, sugere-se uma abordagem individualizada, levando-se em consideração alguns aspectos fundamentais na determinação do alvo glicêmico. Dentre os fatores que justificam um controle menos intensivo destacam-se: história longa de doença, presença de comorbidades, disfunção renal, baixa expectativa de vida, complicações vasculares estabelecidas e fatores associados a maior risco de hipoglicemia (cognição, alfabetismo funcional e suporte social)..$^{30}$

Com base nesses resultados, as Sociedades Médicas têm se posicionado, a fim de que sejam estabelecidas metas para o controle glicêmico em idosos, conforme demonstrado no Quadro 1.

De acordo com a American Geriatrics Society (AGS), um alvo glicêmico com $\mathrm{HbA} 1 \mathrm{C}$ entre $7,5 \%$ e $8 \%$ deve ser buscado nos idosos saudáveis com expectativa de vida maior que 10 anos, podendo ser reduzido para $7 \%$ a 7,5\%, a depender das condições clínicas e sociais de cada um. Ou seja, para os indivíduos autônomos, independentes e com poucas comorbidades, recomenda-se 
um controle glicêmico mais intensivo. Por outro lado, para aqueles que possuem maior número de complicações relacionadas ao diabetes, incapacidades, maior tempo de doença e menor expectativa de vida, a meta da $\mathrm{HbA} 1 \mathrm{C}$ pode ser elevada para níveis entre $8 \%$ e $9 \% .{ }^{31}$ Deve-se ressaltar também que não existem evidências para a utilização de medicações hipoglicemiantes, como sulfonilureias e insulinas, buscando medidas de $\mathrm{HbA} 1 \mathrm{C}$ menores que $6,0 \%$, dado que valores abaixo dessa meta estão associados à hipoglicemia e ao aumento de mortalidade. ${ }^{27}$

Outras sociedades também se posicionaram, a fim de estabelecer metas para controle glicêmico. A American Diabetes Association (ADA) sugere um alvo com $\mathrm{HbA} 1 \mathrm{C}$ de $7,5 \%, 8 \%$ ou $8,5 \%$, a depender das características de cada indivíduo. ${ }^{6}$ Para aqueles idosos saudáveis, com poucas comorbidades, independentes, autônomos e expectativa de vida longa, é sugerido um controle mais intenso, enquanto que recomenda-se um controle menos agressivo para os idosos com multimorbidades, doença renal crônica terminal, alteração cognitiva moderada a grave, alteração em mais de 2 atividades instrumentais de vida diárias ou baixa expectativa de vida.

A International Diabetes Federation (IDF) definiu alvos glicêmicos de acordo com a capacidade funcional, comorbidades e doença cardiovascular estabelecida, sendo sugeridos valores individualizados, levando em consideração o risco de hipoglicemia. Para aqueles indivíduos independentes na realização de atividades de vida diária, com mínima necessidade de suporte, sem comorbidades importantes (categoria 1), sugere-se um alvo de $\mathrm{HbA} 1 \mathrm{C}$ entre $7,0 \%$ e $7,5 \%$. Por outro lado, para aqueles com dependência para realizar atividades de vida diária, com maior necessidade de cuidados médicos e suporte social (categoria 2), recomenda-se um alvo de $\mathrm{HbA} 1 \mathrm{C}$ maior. Uma vez que essa categoria inclui um grande espectro de idosos dependentes, foram estabelecidas 2 subcategorias (A-Síndrome de fragilidade, B-Demência), nas quais é preciso ter maior atenção para a segurança do paciente, pelo maior risco de hipoglicemia. ${ }^{32}$

A European Association for the Study of Diabetes (EASD) indica um alvo de 7,0 a $7,5 \%$ para indivíduos sem comorbidades maiores e 7,6 a 8,5\% para indivíduos frágeis, com perda de independência, multimorbidades, moradores de instituição de longa permanência, incluindo aqueles com demência, com alto risco de hipoglicemia, que tenham pouco benefício no tratamento agressivo. ${ }^{30,33,34}$

A American Association of Clinical Endocrinologists e o American College of Endocrinology (AACE/ACE) tam- bém orientam que o alvo glicêmico deva ser individualizado com base em fatores como idade, expectativa de vida, comorbidades, duração do diabetes, risco de hipoglicemia, motivação do paciente e adesão. Com base nesses critérios, indicam um alvo de $\mathrm{HbA1C} \leq 6,5 \%$ para os indivíduos saudáveis, sem comorbidades importantes, com baixo risco de hipoglicemia e $>6,5$ para aqueles com comorbidades mais graves e risco maior de hipoglicemia. ${ }^{35}$

Com base nas metas individualizadas estabelecidas para cada paciente, pode-se lançar mão de estratégias farmacológicas de tratamento que incluem os antidiabéticos orais e insulinas. Ao tratar o idoso é preciso ter cuidado com o uso de sulfonilureias de meia vida longa (clorpropamida, eglibenclamida), tiazolidinedionas em pacientes com insuficiência cardíaca ou osteoporose e insulinas sem dose fixa, que acarretam em maior risco de hipoglicemia. ${ }^{17}$ Outra questão importante é a correção de dose para algumas drogas de acordo com a função renal, a exemplo da metformina, sulfonilureias, inibidores da DPP4 (com exceção da linagliptina) e insulina. É preciso sempre buscar a terapêutica mais adequada, de forma a evitar hipoglicemias. Naqueles indivíduos com indicação de insulina, o uso de análogos de meia vida longa parece ser mais seguro, assim como a utilização de canetas, uma vez que a dose pode ser calculada de acordo com o "click" emitido pelo dispositivo. ${ }^{36}$ Essa estratégia parece reduzir a chance de erros de posologia, principalmente nos indivíduos com baixo alfabetismo em saúde, déficits sensoriais e suporte social inadequado.

Quadro 1. Metas de controle glicêmico de acordo com as diversas sociedades médicas.

Meta de $\mathrm{HbA} 1 \mathrm{C}$

ADA, 2018

\begin{tabular}{ll}
\hline \hline Idosos saudáveis, com poucas doenças & $<7,5 \%$ \\
crônicas, independentes e autônomos, & \\
expectativa de vida longa. & \\
\hline \hline Idosos com 3 ou mais doenças crônicas, & $<8 \%$ \\
ou alteração em mais de 2 atividades & \\
instrumentais de vida diárias, ou & \\
alteração cognitiva leve a moderada. & \\
Expectativa de vida moderada com alto & \\
risco de hipoglicemia, risco de quedas e & \\
vulnerabilidade. & \\
\hline Idosos com comorbidade grave que afeta & $<8,5 \%$ \\
de forma considerável a saúde, alteração \\
cognitiva moderada a grave, ou alteração em \\
mais de 2 atividades instrumentais de vida \\
diárias, baixa expectativa de vida.
\end{tabular}




\begin{tabular}{ll}
\hline \hline AGS, 2013 & \\
\hline \hline $\begin{array}{l}\text { Pode ser apropriado em indivíduos com } \\
\text { poucas comorbidades, independente para } \\
\text { atividades de vida diárias, com baixo risco de } \\
\text { hipoglicemia. }\end{array}$ & \\
\hline \hline $\begin{array}{l}\text { Valor sugerido para meta de controle em } \\
\text { idosos de forma geral. }\end{array}$ & $7,5-8 \%$ \\
\hline \hline $\begin{array}{l}\text { Indivíduos com múltiplas comorbidades, } \\
\text { saúde precária e baixa expectativa de vida. }\end{array}$ & $8-9 \%$ \\
\hline \hline \begin{tabular}{l} 
IDF, 2013 \\
\hline Categoria 1: indivíduos independentes para \\
realizar atividade de vida diária, com mínima \\
necessidade de suporte, sem comorbidades \\
importantes.
\end{tabular} \\
\hline \hline
\end{tabular}

Categoria 2: indivíduos com dependência para realizar atividades de vida diária, com maior necessidade de cuidados médicos e suporte social. Essa categoria inclui um grande espectro de idosos dependentes, devendo ser destacadas 2 subcategorias, nas quais é preciso ter maior atenção para a segurança do paciente, pelo maior risco de controle inadequado e hipoglicemia:

- Subcategoria A: síndrome de fragilidade que inclui fadiga, perda de peso,dificuldade de mobilidade, risco de quedas e de institucionalização (Fried et. al., 2001).

- Subcategoria B: demência que leva a uma dificuldade para o autocuidado.

Meta de $\mathrm{HbA} 1 \mathrm{C}$

Categoria 3: cuidados em fim de vida que caracterizam indivíduo com doença grave ou neoplasia com expectativa de vida menor

Evitar hiperglicemia sintomática que 1 ano.

\begin{tabular}{ll}
\hline \hline EASD, 2011 & \\
\hline \hline Indivíduos sem comorbidades maiores. & $7-7,5 \%$ \\
\hline \hline Indivíduos frágeis, com perda de & $7,6-8,5 \%$ \\
independência, multimorbidades, moradores \\
de instituição de longa permanência, \\
incluindo aqueles com demência, com alto \\
risco de hipoglicemia, com baixo benefício \\
no tratamento agressivo. \\
\hline \hline AACE/ACE, 2018 \\
\hline \hline $\begin{array}{l}\text { Indivíduos saudáveis, sem comorbidades } \\
\text { importantes, com baixo risco de } \\
\text { hipoglicemia. }\end{array}$ \\
\hline \hline $\begin{array}{l}\text { Indivíduos com comorbidades mais graves e } \\
\text { risco maior de hipoglicemia. }\end{array}$ \\
\hline \hline
\end{tabular}

$\mathrm{HbA1C}=$ Hemoglobinaglicada; $\mathrm{ADA}=$ American Diabetes Association; AGS = American Geriatrics Society; IDF = International Diabetes Federation; EASD = European Association for the Study of Diabetes; AACE/ACE = American Association of Clinical Endocrinologists/American College of Endocrinology.

\section{CONCLUSÕES}

Esta revisão buscou trazer características pertinentes na apresentação do diabetes no idoso, de forma a esclarecer aspectos que interferem no diagnóstico e tratamento. É preciso que essa população seja abordada de forma diferenciada, devendo sempre individualizar cada paciente de acordo com sua capacidade funcional, autonomia, presença de comorbidades e fatores de risco para hipoglicemia, a fim de se proporcionar tratamento adequado. Dadas as evidências atuais, a meta glicêmica deve sempre ser estabelecida de forma a evitar desfechos negativos.

\section{REFERÊNCIAS}

1. Fonte: http://www.idf.org/diabetesatlas, acessado 30 de abril de 2018.

2. Hu FB, Leitzmann MF, Stampfer MJ, Colditz GA, Willett WCet al. Physical activity and television watching in relation to risk for type 2 diabetes mellitus in men. Arch Intern Med. 2001a; 161: 1542-1548.

3. Hu FB, Manson JE, Stampfer MJ, Colditz G, Liu Set al. Diet, lifestyle, and the risk of type 2 diabetes mellitus in women. N Engl J Med. 2001b; 345: 790-797.

4. Fonte: http://portalsaude.saude.gov.br/images/ pdf/2014/abril/30/Lancamento-Vigitel-28-04-ok.pdf, acessado em maio de 2018.

5. Sociedade Brasileira de Diabetes. Diretrizes da Sociedade Brasileira de Diabetes. 2015-2016.

6. American Diabetes Association - ADA. Standards of Medical Care in Diabetes. Diabetes Care. 2018; 41.

7. Kirkman MS, Briscoe VJ, Clark N, Florez H, Haas LB et al. Diabetes in older adults: a consensus report. J Am Geriatr Soc. 2012; 60(12): 2342-56.

8. Fried LP, Tangen CM,Walston J, Newman AB, Hirsch $C$ et al. Journals of Gerontology Series AbiologicalSciences\& Medical Sciences. 2001; 56(3): 146-156.

9. Nouwen A, Winkley K, Twisk J, Lloyd CE, Peyrot M et al. Type 2 diabetes mellitus as a riskfactor for theonsetofdepression: a systematic review and meta-analysis. Diabetologia. 2010; 53:2480-2486.

10. Papelbaum M, Moreira RO, Coutinho W, Kupfer $R$, Zagury Let al.Depression, glycemic control and type 2 diabetes. DiabetolMetabSyndr. 2011; 3: 26.

11. Xu WL, Von Strauss E, Qu CX, Winblad B, Fratiglioni L. Uncontrolled diabetes increasestheriskofAlzhei mer'sdisease: a population-basedcohortstudy. Diabetologia 2009;52:1031-1039.

12. Hanlon JT, Schmader KE,Koronkowski MJ,Weinberger M,Landsman PBet al.Adverse drug events in high risk older outpatients.J Am Geriatr Soc. 1997; 45: 945-948. 
13. Lazarou J, Pomeranz BH, Corey PN: Incidence of adverse drug reactions in hospitalised patients: a meta-analysis of prospective studies. JAMA. 1998;279: 1200-5.

14. Neumiller JJ, Setter SM. Pharmacologic management of the older patient with type 2 diabetes mellitus. Am J GeriatrPharmacother. 2009; 7: 324-42

15. Kennerfalk A, Ruigómez A,Wallander MA, Wilhelmsen L, Johansson S.GeriatricDrugTherapyand Health CareUtilization in the United kingdom. Ann Pharmacother. 2002; 36(5):797-803.

16. Colagiuri R, Girgis S, Eigenmann C, Gomez M, Griffiths R. National evidenced based guideline for patient education in type 2 diabetes. Canberra: Diabetes Australia and the NHMRC; 2009. Disponivel em: http://static. diabetesaustralia.com.au/s/fileassets/diabetes-australia/ b9b8789d-c7ba-473d-bd49-0b7d793a0835.pdf.

17. American GeriatricsSociety.J AmGeriatr Soc. 2015;63(11):2227.

18. Passarelli MCG, Jacob-Filho W, Figueras A.DrugsAging. 2005; 22 (9): 767-777.

19. Geller Al, Shehab N, Lovegrove MC, Kegler SR, Weidenbach KNet al. National estimates of insulin-related hypoglycemia and errors leading to emergency department visits and hospitalizations. JAMA Intern Med. 2014;174(5):678-86.

20. SchwartzAV, Vittinghoff E, Sellmeyer DE,Feingold $\mathrm{QR}$, Rekeneire $\mathrm{N}$ et al.Diabetes-relatedcomplications, glycemiccontrol, andfalls in olderadults. Diabetes Care. 2008; 31(3): 391-396.

21. Zammitt NN, Frier BM. Hypoglycemia in type2 diabetes: pathophysiology, frequency, andeffectsofdifferenttreatmentmodalities. Diabetes Care. 2005; 28:2948-2961.

22. Matyka K, Evans M, Lomas J, Cranston I, Macdonald let al. Altered hierarchy of protective responses against severe hypoglycemia in normal aging in healthy men. Diabetes Care. 1997;20(2):135.

23. Feinkohl I, Aung PP, Keller M, Robertson CM, Morling JR et al. Severe hypoglycemia and cognitive decline in older people with type 2 diabetes: the Edinburgh Type 2 Diabetes Study. Diabetes Care 2014;37:50-515.

24. Dagogo-Jack Samuel. Pitfalls in the use ofHbA1c as a diagnostictest: theethnicconundrum. NatureReviews I Endocrinology. 2010; 6: 589-593.

25. UK Prospective Diabetes Study (UKPDS) Group. Intensive blood-glucose control with sulphonylureas or insulin compared with conventional treatment and risk of complications in patients with type 2 diabetes (UKPDS 33). Lancet. 1998; 352: 837-53.

26. Holman RR, Paul SK, Bethel A, Matthews DR, Neil HAW. 10-Year Follow-up of Intensive Glucose Control in
Type 2 Diabetes.N Engl J Med 2008; 359: 1577-89.

27. ACCORD Study Group, Gerstein HC, Miller ME, Byington RP Goff DC et al. Effects of intensive glucose lowering in type 2 diabetes. N Engl J Med 2008. 358(24): 2545-2559.

28. ADVANCE CollaborativeGroup, Patel A, MacMahon S, Chalmers J, Neal B et al. Intensive blood glucose control and vascular outcomes in patients with type 2 diabetes. N Engl J Med2008. 358(24): 2560-2572.

29. Duckworth W, Abraira C, Moritz T, Reda D, Emanueke Net al; VADT Investigators. Glucose control and vascular complications in veterans with type 2 diabetes. N Engl J Med. 2009; 360(2):129-39.

30. Inzucchi SE, Bergenstal RM, Buse JB, Diamant $M$, Ferrannini Eet al. Management of hyperglycaemia in type 2 diabetes, 2015: a patient-centred approach. Update to a Position Statement of the American Diabetes Association and the European Association for the Study of Diabetes. Diabetologia. 2015; 58: 429-442.

31. American Geriatrics Society - AGS.J Am Geriatr Soc. 2013; 61: 2020-2026.

32. International diabetes federation. Managing older people with type 2 diabetes Global guideline 2013. Disponível em: www.idf.org.

33. Sinclair AJ, Paolisso G, Castro M, Bourdel-Marchasson I, Gadsby Roger, Mañas LR et al. European Diabetes Working Party for Older People 2011 clinical guidelines for type 2 diabetes mellitus. Executive summary. Diabetes Metab. 2011; 37: 27-38.

34. Sinclair A, Morley JE, Rodriguez-Manas L, Paolisso G,Bayer Tet al. Diabetes mellitus in older people: position statement on behalf of the International Association of Gerontology and Geriatrics (IAGG), the European Diabetes Working Party for Older People (EDWPOP), and the International Task Force of Experts in Diabetes. J Am Med Dir Assoc. 2012; 13: 497-502.

35. American Association of Clinical Endocrinologists and American College Of Endocrinology on the comprehensive (AACE/ACE). Consensus statement by the American Association of Clinical Endocrinologists and American College of Endocrinology on the comprehensive Type 2 Diabetes management algorithm-Executive Summary. EndocrPract. 2018; 24(1): 91-120.

36. Rosenstock J,Dailey G, Massi-Benedetti M, Fritsche A,Lin Z et al. Diabetes Care. 2005; 28: 950 -955.

1- Serviço de Geriatria do HSI.

Endereço para correspondência:

jonas.gordilho@gmail.com 